\title{
Similarity Solutions of Two Dimensional Turbulent Boundary Layers by Prandtl Mixing Length Modelling
}

\author{
Bo-Hua Sun $^{1}$ \\ ${ }^{1}$ School of Civil Engineering 83 Institute of Mechanics and Technology \\ Xian University of Architecture and Technology, Xian 710055, China \\ email: sunbohua@xauat.edu.cn \\ (Dated: Jan. 31, 2022)
}

\begin{abstract}
The exact similarity solutions of two dimensional laminar boundary layer were obtained by Blasius in 1908, however, for two dimensional turbulent boundary layers, no similarity solutions (special exact solutions) have ever been found. In the light of Blasius' pioneer works, we extend Blasius similarity transformation to the two dimensional turbulent boundary layers, and successfully transform the two dimensional turbulent boundary layers partial differential equations into a single ordinary differential equation. By author's Maple code, we numerically solve the ordinary differential equation and produce some useful quantities.
\end{abstract}

Keywords: Turbulent boundary layers, laminar boundary layers, similarity transformation, similarity solution, Prandtl mixing length, Reynolds number

\section{INTRODUCTION}

The theory of laminar boundary layer (as shown in Fig.1) dates back to almost 120 years ago [1, 2]. However, in 1921, Prandtl discovered that almost all boundarylayer movements in nature are turbulent rather than laminar [3-8]. The understanding of fully developed turbulence remains a major unsolved problem in classic physics [9-19].

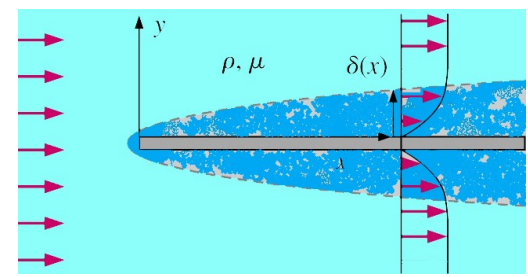

FIG. 1: Turbulent boundary layer.

For the laminar boundary layers, Blasius introduced a similarity transformation and found its solution [2]. The corresponding turbulent problem, i.e., the $2 \mathrm{D}$ turbulent boundary layers have been studied intensively [3, 4] by numerical integrations. Although [9] proposed a similarity solutions for the free shear turbulent flows, such jets. Since those free flows have no fixed walls, it means that the free shear turbulent flows are not the turbulent boundary layers flows. Therefore, to the best of the author's knowledge, no similarity solutions, which are called as special exact solutions by [4], have even been obtained. The question is if the similarity transformation that used to solve the 2D laminar boundary layers could still be extended to the $2 \mathrm{D}$ turbulent boundary layers. If it were possible, how to formulate and what conditions must be hold. Those questions remain open.
In this paper, in the light of Blasius's pioneer work, we extend Blasius similarity transformation to the two dimensional turbulent boundary layers, successfully transform the two dimensional turbulent boundary layers partial differential equations into a single ordinary differential equation. To solve and numerically solve the ordinary differential equation, the author wrote a Maple code.

After introduction in Section 1, the rest of this paper is organized as follows. In Section 2, we formulate the 2D turbulent boundary layers and introduce a similarity transformation. Under special conditions, the partial differential equations of the $2 \mathrm{D}$ turbulent boundary layers can be reduced to a single ordinary differential equation. In Section 3, we write a Maple code to numerically solve the ordinary differential equation. In Section 4, some useful quantities are obtained. Finally, a discussion is presented, and conclusions are drawn. As the essential part of this paper, a Maple code is provided.

\section{FORMULATIONS AND SIMILARITY TRANSFORMATION}

A thin flat plate is immersed at zero incidence in a uniform stream as shown in Fig.1, which flows with speed $U x)$ and is assumed not to be affected by the presence of the plate, except in the boundary layer. The fluid is supposed unlimited in extent, and the origin of coordinates is taken at the leading edge, with $x$ measured downstream along the plate and $y$ perpendicular to it. Assuming that the turbulent flow is steady with pressure gradient along the $x$ axis.

The Reynolds-averaged Navier-Stokes equations [5] of the two dimensional turbulent boundary layers flow un- 
der gradient, $d p / d x$, are reduced to

$$
\begin{aligned}
\frac{\partial \bar{u}}{\partial x}+\frac{\partial \bar{v}}{\partial y} & =0, \\
-\frac{1}{\rho} \frac{\partial p}{\partial y}+\frac{d \bar{v}^{\prime 2}}{d y} & =0, \\
\bar{u} \frac{\partial \bar{u}}{\partial x}+\bar{v} \frac{\partial \bar{u}}{\partial y} & =-\frac{1}{\rho} \frac{d p}{d x}+\nu \frac{d^{2} \bar{u}}{d y^{2}}-\frac{d \overline{u^{\prime} v^{\prime}}}{d y},
\end{aligned}
$$

and boundary conditions:

$$
\begin{aligned}
& y=0: \bar{u}=\bar{v}=0, u^{\prime}=0, v^{\prime}=0 \\
& y=\delta(x): \bar{u}=U(x), u^{\prime}=0, v^{\prime}=0
\end{aligned}
$$

where $\bar{u}$ is the mean velocity, $\nu$ is the kinematic viscosity, $\rho$ is flow density, $p$ is pressure, $u^{\prime}$ and $v^{\prime}$ are velocity fluctuation component, $U(x)$ is outer of boundary layer potential flow velocity. The pressure gradient must be negative, namely $d p / d x<0$, to maintain the flow motion.

Integration of Eq.2 yields

$$
\overline{v^{\prime 2}}+\frac{p}{\rho}=\frac{p_{e}}{\rho},
$$

where $p_{e}$ is a function of $x$ only [3]. Because $\overline{v^{\prime 2}}$ can be neglected comparing with pressure $p$, then $\partial p / \partial x \approx$ $d p_{0} / d x$. From Bernoulli equation, we have relation: $p_{e}+$ $\frac{1}{2} \rho U^{2}=$ constant., leads to $\frac{d p_{e}}{d x}=\rho U \frac{d U}{d x}$. The boundary equations are reduced to following:

$$
\begin{aligned}
\frac{\partial \bar{u}}{\partial x}+\frac{\partial \bar{v}}{\partial y} & =0 \\
\bar{u} \frac{\partial \bar{u}}{\partial x}+\bar{v} \frac{\partial \bar{u}}{\partial y} & =U \frac{d U}{d x}+\nu \frac{d^{2} \bar{u}}{d y^{2}}-\frac{d \overline{u^{\prime} v^{\prime}}}{d y}
\end{aligned}
$$

Introducing a stream function $\Psi(x, y)$ and express the velocity components as follows

$$
\bar{u}=\frac{\partial \Psi}{\partial y}, \quad v=-\frac{\partial \Psi}{\partial x}
$$

with the relation in Eq.9, the mass conservation Eq. 7 is satisfied, and the momentum conservation Eq. 8 becomes

$$
\frac{\partial \Psi}{\partial y} \frac{\partial \Psi}{\partial x \partial y}-\frac{\partial \Psi}{\partial x} \frac{\partial \Psi}{\partial y^{2}}=U \frac{d U}{d x}+\nu \frac{d^{3} \Psi}{d y^{3}}-\frac{d \overline{u^{\prime} v^{\prime}}}{d y}
$$

According to Blasius laminar boundary similarity theory $[2,4]$, the system has no characteristic length, we can assume that the velocity profiles at different distances from the leading edge are affine or similarity to one another, i.e. that the velocity profile $\bar{u}$ at different distances $x$ can be mapped onto each other by suitable choice of scaling factors for $\bar{u}$ and $y$. A suitable scaling factor for $u$ could be the free stream velocity $U(x)$, while for $y$, "boundary-layer thickness" $\delta(x)$, which increases with distance $x$, could be used. The similarity law of the velocity profile can thus be written as $u /[\delta(x) U]=f(\eta)$ with $\eta=y / \delta(x)$, where the function $f(\eta)$ is independent of $x$.

Introducing following transformations

$$
\begin{aligned}
\Psi & =U(x) \delta(x) f(\eta) \\
\eta & =\frac{y}{\delta(x)}
\end{aligned}
$$

thus the velocity components become

$$
\begin{aligned}
& \bar{u}=U \frac{d f}{d \eta} \\
& \bar{v}=-\left(\delta f \frac{d U}{d x}+U f \frac{d \delta}{d x}-\eta U \frac{d \delta}{d x} \frac{d f}{d \eta}\right) .
\end{aligned}
$$

Substituting Eqs.13 and 14 into Eq. 11, we have

$$
\frac{d^{3} f}{d \eta}+\alpha f \frac{d^{2} f}{d \eta^{2}}+\beta\left[1-\left(\frac{d f}{d \eta}\right)^{2}\right]-\frac{\delta}{\nu U} \frac{d}{d \eta} \overline{u^{\prime} v^{\prime}}=0,
$$

where the coefficients are

$$
\alpha=\frac{\delta}{\nu} \frac{d U \delta}{d x}, \quad \beta=\frac{\delta^{2}}{\nu} \frac{d U}{d x} .
$$

According to the Prandtl mixing length theory [8], the Reynolds stress is proposed to be

$$
\tau_{x y}^{\prime}=-\rho \overline{u^{\prime} v^{\prime}}=\rho \ell^{2}\left|\frac{d \bar{u}}{d y}\right| \frac{d \bar{u}}{d y},
$$

where $\bar{u}$ is the mean velocity, $\rho$ is flow density, $u^{\prime}$ and $v^{\prime}$ are velocity fluctuation component. The mixing length $\ell$ must have length scale who should be a form that satisfy the boundary condition $\overline{u^{\prime} v^{\prime}}=0$ at both bottom $(y=0)$, hence Prandtl proposed the mixing length as follows

$$
\ell=\kappa y,
$$

where $\kappa \approx 0.4$ is the von Kármán constant. Hence we have

$$
\overline{u^{\prime} v^{\prime}}=-\ell^{2}\left|\frac{d \bar{u}}{d y}\right| \frac{d \bar{u}}{d y}=-(\kappa y)^{2}\left|\frac{d \bar{u}}{d y}\right| \frac{d \bar{u}}{d y},
$$

with the Eqs.13 and 14, the above relation can expressed as follows

$$
\overline{u^{\prime} v^{\prime}}=-\kappa^{2} U^{2} \eta^{2}\left|\frac{d^{2} f}{d \eta^{2}}\right| \frac{d^{2} f}{d \eta}
$$

hence Eq.15 can be reduced to

$\frac{d^{3} f}{d \eta}+\alpha f \frac{d^{2} f}{d \eta^{2}}+\beta\left[1-\left(\frac{d f}{d \eta}\right)^{2}\right]+\frac{\kappa^{2} U \delta}{\nu} \frac{d}{d \eta}\left(\eta^{2} \frac{d^{2} f}{d \eta^{2}}\left|\frac{d^{2} f}{d \eta^{2}}\right|\right)=0$ 
and its three boundary conditions:

$$
\begin{aligned}
& \eta=0: f=0, \frac{d f}{d \eta}=0, \\
& \eta=1: \frac{d f}{d \eta}=1 .
\end{aligned}
$$

General speaking, the coefficients are the functions of $x$, then there is no similarity solution. However, in the 3rd term of Eq.21, if set $U \delta$ as a constant, ie., $U \delta=C$, leads $\alpha=0$ and

$$
\beta=\frac{1}{\nu} \delta\left(\frac{d \delta U}{d x}-U \frac{d \delta}{d x}\right)=-\frac{1}{\nu} U \delta \frac{d \delta}{d x}=\frac{C}{\nu} \frac{d \delta}{d x},
$$

if the coefficient $\beta$ is assumed to be constant, then we have the function $\delta$ as follows $\delta(x)=\nu \frac{\beta}{C} x+B$, where $B$ is an integration constant. Since $\delta(0)=\delta_{0}$, thus $B=\delta_{0}$, hence we have

$$
\delta(x)=\nu \frac{\beta}{C} x+\delta_{0}
$$

With the obtained $\delta(x)$ we have $U(x)=\frac{C}{\delta(x)}$. Set $U(x)=$ $U_{0}$ is the velocity at $x=0$, then we have $C=U_{0} \delta_{0}$, hence

$$
\delta(x)=\delta_{0} A(x), \quad U(x)=\frac{U_{0}}{A(x)},
$$

where $A(x)=\left(1+\frac{\nu \beta}{U_{0} \delta_{0}^{2}} x\right)$.

According to [4], $U(x)=U_{0} / A(x)$, which is the outside the boundary layer for convergent $(\beta<0)$ or divergent $(\beta>0)$ radial motion between intersecting planes, respectively. The negative and positive sign indicates that the motion is towards to and away from the line of intersection. The laminar boundary layer motion due to convergent flow was discussed by Pohlhausen (1921) [20].

Finally, we have successfully transformed the partial differential equations in Eq.10 and its boundary conditions into a single ordinary differential equation as follows

$$
\frac{d^{3} f}{d \eta^{3}}+\beta\left[1-\left(\frac{d f}{d \eta}\right)^{2}\right]+\kappa^{2} \frac{d}{d \eta}\left(\eta^{2} \frac{d^{2} f}{d \eta^{2}}\left|\frac{d^{2} f}{d \eta^{2}}\right|\right)=0
$$

and boundary conditions:

$$
\begin{aligned}
& \eta=0: f=0, \frac{d f}{d \eta}=0, \\
& \eta=1: \frac{d f}{d \eta}=1 .
\end{aligned}
$$

Up to now, there is only parameter $\beta$ still to be given. For different constant $\beta$, the corresponding similarity solutions can be obtained from Eq.27. The constant $\beta$ is a dominate parameter, which directly influence the property of the unknown function $f(\eta)$. Without loss generality and same time for simplicity, we set $\beta=1$ in all our numerical studies.

\section{SERIES SOLUTION AND NUMERICAL STUDIES}

If the boundary layer partial differential equations can be reduced to a single ordinary differential equation, similarity solution can be found. This similarity can further be related to different coordinate planes, $(x, y)$ or $(x, \eta)$, depending on which of the two coordinates transformations treated in the last section. We therefore have the following possibilities for similarity solutions.

Series solutions: The series solution can be expressed as : $f(\eta)=\sum_{n=0}^{\infty} a_{n+2} \eta^{n}$, we have $a_{0}=0, a_{1}=0$, hence $f(\eta)=\sum_{n=2}^{\infty} a_{n} \eta^{n}$. Substituting it into Eq.27 and to determine coefficients under boundary condition. We are not going to solve the problem in the series owing to its poor convergency, we will solve it numerically.

Numerical solutions: A complete Maple code for solving the Eq.27 under the boundary conditions Eqs. 28 and 29 is provided below:

restart; with(student); with(plots); beta $:=1$; kappa $:=0.4$; lambda $:=1 ;$ ode $:=\operatorname{diff}(f(x i), x i, x i, x i)+\operatorname{beta}^{*}(1-\operatorname{diff}(f(x i)$, $\mathrm{xi}) *(1-\operatorname{diff}(\mathrm{f}(\mathrm{xi}), \mathrm{xi}))+$ kappa*kappa*diff( $\mathrm{xi}^{*} \mathrm{xi}^{*} \operatorname{abs}(\operatorname{diff}(\mathrm{f}(\mathrm{xi}), \mathrm{xi}$, $\left.\mathrm{xi}))^{*} \operatorname{diff}(\mathrm{f}(\mathrm{xi}), \mathrm{xi}, \mathrm{xi}), \mathrm{xi}\right)=0$; sol $:=\operatorname{dsolve}($ ode, $\mathrm{f}(0)=0, \mathrm{D}(\mathrm{f})(0)$ $=0, \mathrm{D}(\mathrm{f})($ lambda $)=1$, numeric, output = listprocedure $) ; \mathrm{p} 0:=$ plots:-odeplot(sol, $[\mathrm{xi}, \mathrm{f}(\mathrm{xi})], \mathrm{xi}=0$.. lambda, color $=$ black, linestyle $=[1]$, thickness $=3$, legend $=[\mathrm{f}(\mathrm{xi})]$, axes $=$ boxed $) ; \mathrm{p} 1$ $:=$ plots:-odeplot(sol, $[\mathrm{xi}, \operatorname{diff}(\mathrm{f}(\mathrm{xi}), \mathrm{xi})], \mathrm{xi}=0$.. lambda, color $=$ blue, linestyle $=[2]$, thickness $=3$, legend $=[" \mathrm{df} / \mathrm{dxi} "]$, axes $=$ boxed); p2 $:=$ plots:-odeplot(sol, [xi, $\operatorname{diff}(\mathrm{f}(\mathrm{xi}), \mathrm{xi}, \mathrm{xi})], \mathrm{xi}=0$ .. lambda, color $=$ red, linestyle $=[3]$, thickness $=3$, legend $=$ ["d(df/dxi; $) / d x i "]$, axes = boxed); u := plots:-odeplot(sol, [xi, dif$\mathrm{f}(\mathrm{f}(\mathrm{xi}), \mathrm{xi})], \mathrm{xi}=0 .$. lambda, color $=$ red, linestyle $=[1]$, thickness $=3$, legend $=[" \mathrm{df} / \mathrm{dxi} "]$, axes $=$ boxed $) ; \mathrm{v}:=\operatorname{plots:-odeplot}(\mathrm{sol}$, $[x i, x i * \operatorname{diff}(f(x i), x i)], x i=0$.. lambda, color $=$ blue, linestyle $=$ [3], thickness $=3$, legend $=[" x i ; d f / d x i "]$, axes $=$ boxed); plots:$\operatorname{display}([\mathrm{p} 0, \mathrm{p} 1, \mathrm{p} 2]$, axes $=$ boxed $)$; plots:-display $([\mathrm{u}, \mathrm{v}]$, axes $=$ boxed);

The $f(\eta), \frac{d f}{d \eta}$ and $\frac{d^{2} f}{d \eta^{2}}$ are depicted in Fig.2.

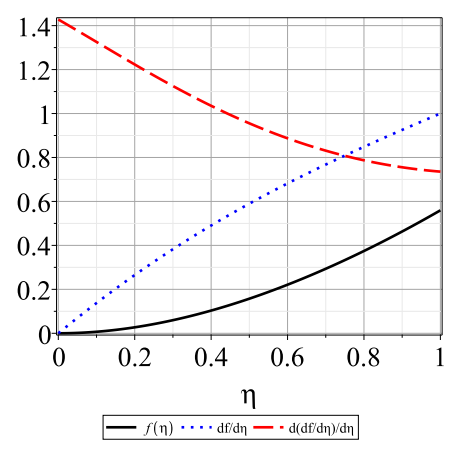

FIG. 2: $f(\eta), \frac{d f}{d \eta}$ and $\frac{d^{2} f}{d \eta^{2}}$, in which $f^{\prime \prime}(0)=\left.\frac{d^{2} f}{d \eta^{2}}\right|_{\eta=0}=1.4278$. 


\section{SOME USEFUL QUANTITIES}

Mean velocity profile: With the $U(x)$ and $\delta(x)$, we have the velocity components as follows

$$
\begin{aligned}
& \bar{u}=\frac{U_{0}}{A(x)} \frac{d f}{d \eta}, \\
& \bar{v}=\frac{\nu \beta}{U_{0} \delta_{0}} \frac{U_{0}}{A(x)} \eta \frac{d f}{d \eta} .
\end{aligned}
$$

For a given distance $x$, the mean velocity profiles $\frac{\bar{u} A(x)}{U_{0}}=$ $\frac{d f}{d \eta}$ and $\frac{U_{0} \delta_{0}}{\nu \beta} \frac{\bar{v} A(x)}{U_{0}}=\eta \frac{d f}{d \eta}$ are depicted in Fig.3.

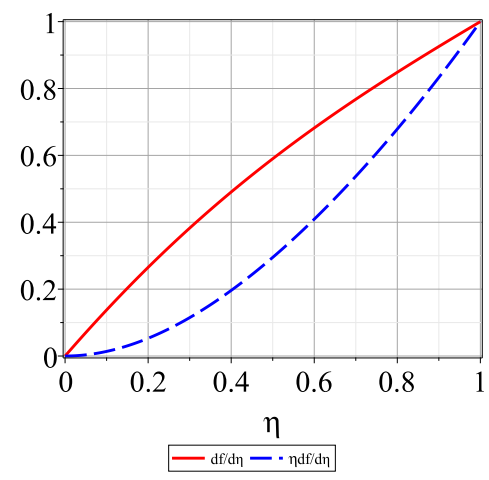

FIG. 3: Mean velocity profile in the case $\beta=1$. The red solid line is $\frac{\bar{u} A(x)}{U_{0}}=\frac{d f}{d \eta}$, and blue dashline is $\frac{U_{0} \delta_{0}}{\nu \beta} \frac{\bar{v} A(x)}{U_{0}}=\eta \frac{d f}{d \eta}$.

Shear stress and drag: Introducing $R e_{0}=\frac{U_{0} \delta_{0}}{\nu}$ and $R e_{x}=$ $\frac{x U_{0}}{\nu}$, the function $A(x)$ becomes

$$
A(x)=1+\beta \frac{R e_{x}}{R e_{0}^{2}} .
$$

The shear stress is given by

$$
\begin{aligned}
\tau_{x y} & =\mu \frac{\partial \bar{u}}{\partial \eta}=\frac{\mu}{\delta(x)} \frac{\partial \bar{u}}{\partial \eta}=\mu \frac{U(x)}{\delta(x)} \frac{d^{2} f}{d \eta^{2}} \\
& =\mu \frac{U_{0}}{\delta_{0}} \frac{1}{A^{2}} \frac{d^{2} f}{d \eta^{2}}=\rho U_{0}^{2} \frac{R e_{0}^{3}}{\left(R e_{0}^{2}+\beta R e_{x}\right)^{2}} \frac{d^{2} f}{d \eta^{2}} .
\end{aligned}
$$

The wall shear stress

$$
\tau_{w}=\tau_{x y}(0)=\rho U_{0}^{2} \frac{R e_{0}^{3}}{\left(R e_{0}^{2}+\beta R e_{x}\right)^{2}} f^{\prime \prime}(0),
$$

The above relation indicates that $\tau_{w} \sim x^{-2}$ decreases when distance $x$ away from the origin.

The drag on one side of a plate of length $L$ and unit breadth is then

$$
D=\int_{0}^{L} \tau_{w} d x=\rho U_{0}^{2} G f^{\prime \prime}(0),
$$

where $G=\frac{\nu}{\beta U_{0}} R e_{0}^{3}\left(\frac{1}{R e_{0}^{2}}-\frac{1}{R e_{0}^{2}+\beta R e}\right)$, and the Reynolds number $R e=\frac{U_{0} L}{\nu}$.

Then we have the drag coefficient is

$$
\begin{aligned}
C_{D} & =\frac{D}{(1 / 2) \rho L U_{0}^{2}}=2 \frac{G}{L} f^{\prime \prime}(0) \\
& =\frac{2}{\beta} \frac{R e_{0}^{3}}{R e}\left(\frac{1}{R e_{0}^{2}}-\frac{1}{R e_{0}^{2}+\beta R e}\right) f^{\prime \prime}(0),
\end{aligned}
$$

The above relation reveals that the drag coefficient is not only dependent on the Reynolds number Re but also thee ratio $R e_{0}$, owing to convergent/divergent nature of the flow.

Velocity fluctuations and total velocity field: In Prandtl mixing-length theory [4, 8], Prandtl assumes a greatly simplified model of the fluctuations, according to which the individual fluid elements are displaced in a mean distance (the mixing length) $\ell$ by the fluctuations, perpendicular to the main flow direction, but still retaining their momentum. The element that was initially at $y$, and is now at $y+\ell$, has a higher velocity than its new surroundings. The velocity difference is a measure of the fluctuation velocity in the $x$ direction:

$$
u^{\prime}=\bar{u}(y+\ell)-\bar{u}(y) \approx \ell \frac{d \bar{u}}{d y} .
$$

Prandtl assumed that the velocity fluctuation component $v^{\prime}$ is the same order of magnitude as $u^{\prime}$, namely, $v^{\prime} \sim u^{\prime}$, and therefore we have

$$
v^{\prime} \sim \ell \frac{d \bar{u}}{d y}
$$

Using the the coordinate transformation 11

$$
\begin{aligned}
& u^{\prime}=U \kappa \eta \frac{d^{2} f}{d \eta^{2}}, \\
& v^{\prime} \sim U \kappa \eta \frac{d^{2} f}{d \eta^{2}},
\end{aligned}
$$

With the mean velocity and fluctuations, we have the total flow velocity components $U$ in $x$ and $y$ directions, respectively:

$$
u=\bar{u}+u^{\prime},
$$

The flow velocity ratios are depicted in Fig.4.

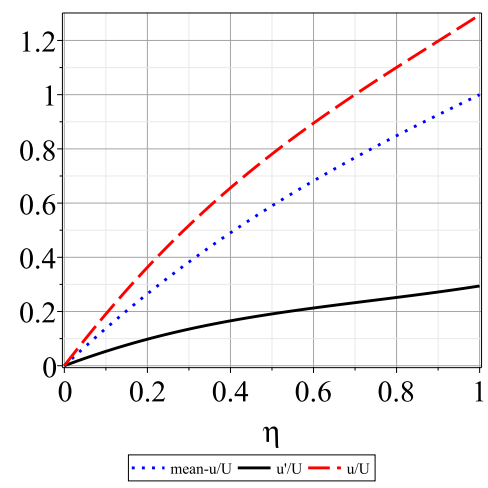

FIG. 4: Flow velocity ratios, in which the blue dot-line is $\bar{u} / U$, black solid line is $u^{\prime} / U$ and red long dashline is $u / U$.

Moment of velocity fluctuation: The square of the streamwise flow velocity fluctuation with the wall distance, i.e., $\overline{\left(u^{\prime}\right)^{2}}$, is given by

$$
\overline{\left(u^{\prime}\right)^{2}}=U^{2} \kappa^{2} \eta^{2}\left(\frac{d^{2} f}{d \eta^{2}}\right)^{2} .
$$

The result in Fig.5 indicate the square of the streamwise flow velocity fluctuation can be approximately expressed in a simple form $\overline{\left(u^{\prime}\right)^{2}} \approx U^{2}\left(0.025 \eta^{2}+0.06 \eta\right)$. 


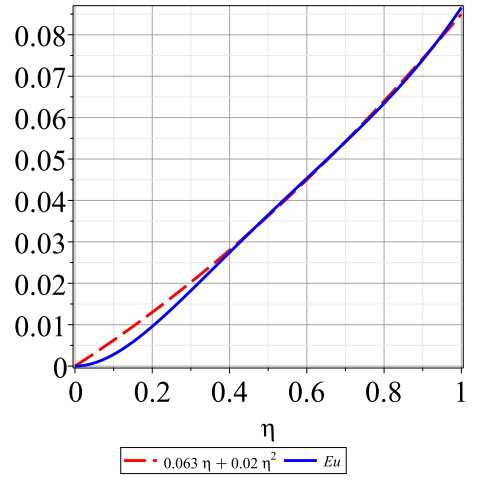

FIG. 5: $E u=\overline{\left(u^{\prime}\right)^{2}} / U^{2}$.

Displacement layer thickness: A physical sensible measure for the thickness of the boundary layer id the displacement thickness $\delta_{u}$. The definition is

$$
\begin{aligned}
\delta_{u} & =\int_{0}^{\delta}\left(1-\frac{\bar{u}}{U}\right) d y \\
& \left.=\delta_{0} A(x) \int_{0}^{1}\left(1-f^{\prime}\right) d\right] d \eta=0.5505 \delta_{0} A(x) .
\end{aligned}
$$

\section{CONCLUSIONS}

To the best of the author's knowledge, with the frame of Prandtl mixing modelling, the similarity solutions that are called as special exact solutions by [4], and all related quantities of the 2D turbulent boundary layers are obtained for the first time. This study may help facilitate a better understanding of turbulence phenomena.

\section{ACKNOWLEDGMENTS}

This work was supported by Xi'an University of Architecture and Technology (Grant No. 002/2040221134).

\section{Interest Conflict}

The author declare that they have no conflict of interest.
[1] L. Prandtl, On fluid motions with very small friction (in german). Third International Mathematical Congress, Heidelberg (1904)

[2] H. Blasius, Grenzschichten in Fljssigkeiten mit kleiner Reibung. Z. Math. Phys., 56(1908) 1-37.

[3] H. Tennekes and J.L. Lumley, A First Course of Turbulence, The MIT Press, Cambridge (1972).

[4] H. Schlichting, Boundary Layer Theory, fourth ed., McGraw-Hills, 1960 translated by J. Kestin.

[5] O. Reynolds, On the dynamical theory of incompressible viscous fluids and the determination of the criterion. Philosophical Transactions of the Royal Society of London. 1895;186, 123-164. https://doi.org/10.1098/rsta.1895.0004

[6] L. Prandtl, Bemerkungen über die entstehung der turbulenz, Z. Angew. Math. Mech. 1(1921):431-436.

[7] Th. von Kármán, Über leminere und turbulence Reibung (On Laminer and Turbulent Friction), Z. Angew. Math. Mech. 1 (1921)223.

[8] L. Prandtl, Bericht über Untersuchungen zur ausgebildeten Turbulenz, Z. Angew. Math. Mech. 5(2)(1925)136139.

[9] A. A. Townsend, The Structure of Turbulent Flow, Cambridge Univ. Press, New York, 1976.

[10] B. Birnir, The Kolmogorov-Obukhov Theory of Turbulence. Springer, New York, 2013.

[11] B. Birnir, The turbulence problem, Nonlinear Studies, 26(4), 2019.

[12] Y. Pomeau and M. Le Berre, Turbulence in a wedge: the case of the mixing layer, Phys. Rev. Fluids 6, 074603 (2021)

[13] Y. Pomeau and M. Le Berre, Turbulent plane Poiseille flow, EPJ, 2021.

[14] B. H. Sun, The temporal scaling laws of compressible turbulence, Modern Physics Letters B, 30(23)(2016) 1650297 (14 pages).

[15] B. H. Sun, Scaling laws of compressible turbulence, Appl. Math. Mech. -Engl. Ed., 38(6)(2017) 765-778.

[16] B. H. Sun, Thirty years of turbulence study in China. Applied Mathematics and Mechanics, 40(2)(2019) 193214.

[17] B. H. Sun, Revisiting the Reynolds-averaged NavierStokes equations, Open Physics 19 (2021) 853-862.

[18] B. H. Sun, Closed form solution of planeparallel turbulent flow along an unbounded plane surface. Preprints (2021) 2021110008 (doi: 10.20944/preprints202111.0008.v3); Fractals - Complex Geometry, Patterns, and Scaling in Nature and Society (accepted).

[19] B. H. Sun, Turbulent Poiseuille flow modelling by an enhanced Prandtl-Driest mixing length. Preprints 2022, 2022010240 (doi: 10.20944/preprints202201.0240.v2). submitted to Applied Mathematics and Mechanics.

[20] K. Pohlhausen, Zur näherungsweisen Integration der Differentialgleichung der laminaren Grenzschicht, Z.angew.Math.Mech.,1,252-268. 